\title{
Efectividad de los ejercicios oculomotores para la insuficiencia de convergencia en adolescentes en tres instituciones educativas en la provincia de Lima
}

Oculomotor effectiveness of exercises for failure of convergence in teenagers in three schools in the province of Lima

\author{
Abraham Alberto Antonio Rojas 1, a, Geraldine Katherine Boza Huamán 1,a,

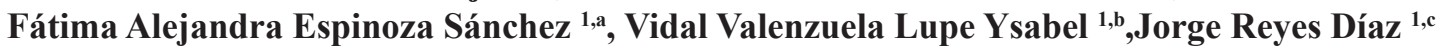

\section{RESUMEN}

La insuficiencia de convergencia es la incapacidad de converger ambos ojos en un mismo punto, genera dificultades en los adolescentes a la hora de realizar las actividades escolares por ello detectarla y tratarla a tiempo es importante. Objetivo: Determinar la efectividad de los ejercicios oculomotores en adolescentes con insuficiencia de convergencia en tres instituciones educativas de Lima.Material y métodos: estudio cuasi-experimental, utilizando como referencia la evaluación oftalmológica antes y después del tratamiento. A los adolescentes que presentaban insuficiencia de convergencia (IC) se les realizo ejercicios oculomotores por un periodo de 1 mes las evaluaciones pre tratamiento y pos tratamiento fue realizada por el oftalmólogo. Resultados: Se evaluaron 321 adolescente de los cuales $225(70,1 \%)$ presentaron IC de ellos 190 cumplieron los criterios de inclusión, solo 56 adolescentes cumplieron la terapia de ejercicios oculomotores, siendo 19 del género femenino $(33,9 \%)$ y 37 del género masculino (66.1\%). La puntuación de la IC con la regla de Krimsky en los adolescentes presento una media del 14,2 $\pm 1,4 \mathrm{~cm}$. para el género femenino y $14,5 \pm 1,43 \mathrm{~cm}$ para el género masculino. La mediana de la puntuación de Punto Proximal de Convergencia (PPC) pre ejercicios oculomotores fue $14 \mathrm{~cm}$ (p25 12,6 cm - p75 15,3cm), y post tratamiento fue 10,6 cm (p25 9,5cm - p75 13,3cm) siendo esta diferencia significativa $(\mathrm{p}<0,05)$. Conclusión: Los ejercicios activos oculomotores en adolescentes disminuyen la insuficiencia de convergencia y puede usarse como tratamiento fisioterapéutico y adyuvante del tratamiento oftalmológico en la IC.

PALABRAS CLAVE: Convergence Insufficiency, Visión Therapy, Orthoptics.

\section{SUMMARY}

Convergence insufficiency is the inability of both eyes to converge at the same point, creates difficulties in adolescents at school activities therefore time detection and treatment is important. Objective: To determine the effectiveness of the oculomotor exercises in adolescents with convergence insufficiency in three educational institutions in Lima. Material and methods: quasi-experimental study, using as reference the ophthalmologic evaluation before and after treatment. Adolescents who had convergence insufficiency (CI) were oculomotor exercises for a period of one month after the pre-treatment evaluation and treatment was performed by the ophthalmologist. Results: 321 adolescents were evaluated, 225 (70.1\%) of them showed IC, 190 met the inclusion criteria, only 56 adolescents

\footnotetext{
Escuela Profesional de Tecnología Médica, Facultad de Medicina Alberto Hurtado, Universidad Peruana Cayetano Heredia. Lima, Perú.

a Egresada; ${ }^{\mathrm{b}}$ Magister en Epidemiologia; ${ }^{\mathrm{c}}$ Doctor
} 
finished therapy oculomotor exercises, being 19 females (33.9\%) and 37 males (66.1\%). The score of the IC with the rule Krimsky in adolescents presented an average of $14.2 \pm 1.4 \mathrm{~cm}$. for females and $14.5 \pm 1.43 \mathrm{~cm}$ for males. The median score of Proximal Point Convergence (CPP) pre oculomotor exercises was $14(12.6 \mathrm{p} 25-\mathrm{p} 7515.3) \mathrm{cm}$. and post treatment was 10.6 (p25 9.5 - p75 13.3) cm difference was significant $(\mathrm{p}<0.05)$. Conclusion: Oculomotor active exercises in adolescents decrease convergence insufficiency and can be used as physical therapy and adjuvant treatment of ophthalmologic.

KEYWORDS: Convergence insufficiency, vision therapy, orthoptics.

\section{INTRODUCCIÓN}

La insuficiencia de convergencia (IC) es una anomalía del sistema visual binocular, es la inhabilidad para obtener o mantener una convergencia adecuada sin esfuerzo. (1) La IC es una disfunción no estrábica de la visión binocular, una condición benigna e idiopática que puede pasar desapercibida repercutiendo en el rendimiento laboral y académico. La etiología de la IC puede ser de origen anatómico, por disturbios en la convergencia acomodativa, enfermedades generales o causas psíquicas. (2) La sintomatología se presenta con fatiga ocular, cefaleas, enrojecimiento ocular y lagrimeo. En algunos pacientes es asintomático. (3)

Los efectos de la IC se reflejan en la motilidad que es la habilidad para mover los ojos de manera precisa, suave y a toda dirección, incluyen, estabilidad de la fijación, movimientos sacádicos y movimientos de seguimiento (4). La acomodación permite ver con nitidez a todas las distancias y realizar cambios bruscos de enfoque. Y, la visión binocular para coordinar adecuadamente la alineación de los dos ojos (5).

Los ejercicios oculotomores están basados en la fisiología (6), anatomía (7), en la neurología de la visión binocular (8) y el desarrollo (9).

En España los estudios están enfocados en la prevalencia y análisis de la eficacia de la terapia para la IC, ya que aún los valores para los signos de diagnóstico son escasos. (10) Un estudio realizado en la Universidad de la Salle de Colombia indica que los estudios realizados en dicho país, están dirigidos a analizar la eficacia de la terapia y buscar los medios de medición para el diagnóstico más eficaz de la IC, además, de presentar avanzados tratamientos oculares con sus respectivos protocolos, cuentan con equipos y baterías diseñadas para cada tratamiento (11). Dos estudios encontrados en la base de datos Pubmed, solo describen ejercicios en casa para el tratamiento de la IC. Un estudio realizado en USA del Nationwide Children's Hospital estima una incidencia de insuficiencia de convergencia de 2,5 $\%$ a 13\% para el 2014 en la población general. (12) Mientras que exodesviaciones están solo presentes en el $1 \%$ de la población general, la insuficiencia de convergencia está presente en $11 \%-19 \%$ de niños con exodesviaciones. (13)

En España el licenciado en fisioterapia Iñaki Pastor, ha desarrollado el diagnóstico y el tratamiento de las alteraciones del sistema oculomotor a través de la terapia manual sobre el globo ocular, las estructuras blandas que lo rodean y sobre los centros neurológicos que controlan su alineación y movimiento coordinado. Se menciona también que la osteopatía fue la primera en abordar los problemas oculares sin una explicación científica. El trabajo que realiza es multidisciplinario, trabaja con oftalmólogos y optómetras, considerando la terapia visual como ayuda sensorial en el tratamiento. (14)

En el mismo país ibérico, fisioterapeutas aplican un tratamiento llamado Terapia Miofascial, abarca un análisis general del control postural con relación al sistema miofascial (15)

En nuestro país las consultas para oftalmología han ido en aumento, es así que el Instituto Nacional de Oftalmología en su anuario del 2010 marca que partir del 2007 han incrementado sus atenciones en $15 \%$ y en el 2008 han aumentado en 20\% respecto al 2006. En el 2010 se han atendido casi de 346.129 pacientes. (16)

El presente trabajo se realizó en estudiantes de nivel secundario que presentan insuficiencia de convergencia produciendo dificultades a la hora de realizar las actividades frente a la computadora o durante la elaboración de sus deberes escolares. La insuficiencia de convergencia es un problema que se presenta con mayor frecuencia en la población, si bien el optómetra está más relacionado con las patologías 
o dificultades visuales mayormente sensitivas, el fisioterapeuta se enfoca en las patologías motoras ya que la cavidad orbitaria presenta músculos que intervienen en los movimientos oculares, por lo que consideramos que los terapeutas físicos como especialistas en el movimiento motor pueden cumplir un papel importante dentro de la rehabilitación visual. El objetivo de este estudio fue determinar la efectividad de los ejercicios oculomotores para la Insuficiencia de Convergencia en adolescentes de tres instituciones educativas de la provincia de Lima.

\section{MATERIAL Y MÉTODOS}

Estudio cuasi experimental pre- post tratamiento de ejercicios oculomotores de un grupo de adolescentes, estudiantes de nivel secundario de las instituciones educativas.

\section{Criterios de inclusión:}

Estudiantes de nivel secundario de las Instituciones Educativas.

Adolescentes que sean emétropes y amétropes con corrección.

Adolescentes con resultado de visión normal en agudeza visual. (20/15 20/20 20/25)

Diagnóstico de IC.

Aceptación del consentimiento $\mathrm{y}$ asentimiento informado.

\section{Criterios de exclusión:}

Adolescentes operados de estrabismo

El procedimiento de muestreo fue no probabilístico por conveniencia se realizó en tres Instituciones educativas: Institución Educativa Particular Giordano Bruno del distrito de Breña, Institución Educativa Scipion Llona del distrito de Miraflores y La Institución Educativa Emblemática Meliton Carvajal del distrito de Lince cada uno con 5 salones de aproximadamente 27 estudiantes en cada uno de ellos, siendo un total de 441 estudiantes. Se realizó una invitación a los adolescentes y los padres de familia para participar en una evaluación oftalmológica. A los que aceptaron, que fueron 321 adolescentes, se les realizó una evaluación oftalmológica; de ellos 225 presentaron insuficiencia de convergencia y solo 190 adolescentes cumplieron los criterios de elección. Se les invitó a formar parte del estudio, dándoles el consentimiento informado a los padres y el asentimiento a los adolescentes. Sólo 60 adolescentes y padres de familia aceptaron participar en el estudio.
Para el cálculo del tamaño de muestra se realizó un piloto de 11 niños, con ello se determinó la varianza de la diferencia de la distancia de PPC de recuperación del antes y después del tratamiento

$$
n=\left[\frac{\left(z_{\alpha}+z_{\beta}\right) * \sigma}{d}\right]^{2}=\left[\frac{(1.95+0.84) * 11.7}{4}\right]^{2}=67
$$

“ $\sigma$ " es la desviación estándar de las diferencias experimentadas por el grupo en los dos momentos estudiados obtenidos por el piloto $(11.7 \mathrm{~cm})$

"d" es la diferencia en las mediciones antes y después del estudio del PPC, se asumió como 4 por criterio del oftalmológico.

" $\mathrm{n}$ " son los individuos necesarios en la muestra, " $\mathrm{Z}$ $\alpha$ "es el valor $\mathrm{z}$ del nivel de significancia estadística, típicamente 1.95 para un nivel de 0.05 , “ $Z \beta$ “es el valor $\mathrm{z}$ del poder deseado típicamente 0.84 para un poder de 0.8 .

Por las dificultades en la captación de los adolescentes al ingreso del estudio solo aceptaron iniciar los ejercicios oculomotores 56 adolescentes. Por ello se calculó la potencia para rechazar la hipótesis nula siendo falsa, con lo cual dio por resultado una potencia de $100 \%$.

\section{Herramienta de medición}

Se realizaron pruebas oftalmológicas como son el PPC agudeza visual y cover test. Estos exámenes estuvieron a cargo del oftalmólogo, el cual dio un diagnostico asegurando si los adolescentes presentaban o no IC.

Los resultados para diagnosticar la insuficiencia de convergencia son (tabla 1):

Punto Proximal de Convergencia: El PPC es la mínima distancia en la cual los ejes ópticos se cruzan en la máxima convergencia para observar un objeto único y sencillo. Se puede evaluar mediante el acercamiento de un haz de luz, objeto real y con luz y filtro rojo. Se consideró solo la evaluación con objeto real (figura de una mariposa de $1 \mathrm{~cm}^{2}$ y un Punto Próximo de Convergencia anómalo mayor $10 \mathrm{~cm}$. El instrumento de medición fue la regla de Krimsky. Según la medición dada se puede clasificar la IC en los siguientes niveles: PPC normal: $<10 \mathrm{~cm}$ PPC Leve: 10 a $14 \mathrm{~cm}$, PPC Moderado: 15 a $22 \mathrm{~cm}$ y PPC Alto: $>23 \mathrm{~cm}$. (17) 
Tabla 1. Herramientas de medición.

\begin{tabular}{|c|c|c|c|c|}
\hline \multicolumn{5}{|c|}{ IDENTIFICACIÓN DE VARIABLES } \\
\hline VARIABLE & $\begin{array}{l}\text { DEFINICIÓN } \\
\text { CONCEPTUAL }\end{array}$ & $\begin{array}{c}\text { DEFINICIÓN } \\
\text { OPERACIONAL }\end{array}$ & $\begin{array}{c}\text { TIPO DE } \\
\text { VARIABLE }\end{array}$ & $\begin{array}{l}\text { INSTRUMENTO } \\
\text { DE MEDIDA }\end{array}$ \\
\hline $\begin{array}{c}\text { PUNTO } \\
\text { PROXIMAL DE } \\
\text { CONVERGENCIA }\end{array}$ & $\begin{array}{l}\text { Mínima distancia en la } \\
\text { cual los ejes ópticos se } \\
\text { cruzan en la máxima } \\
\text { convergencia para } \\
\text { observar un objeto único } \\
\text { y sencillo. }\end{array}$ & $\begin{array}{l}\text { Son ejercicios realizados } \\
\text { con objeto real. Se evaluará } \\
\text { la distancia en centímetros. } \\
\text { Según la medición dada se } \\
\text { puede clasificar la IC en } \\
\text { los siguientes niveles: Leve } \\
\text { entre } 10 \text { a } 14 \mathrm{~cm} \text {, moderado } \\
15 \text { a } 22 \mathrm{~cm} \text { y alto mayor a } \\
23 \text { Examen a realizarlo el } \\
\text { oftalmólogo }\end{array}$ & $\begin{array}{l}\text { Cuantitativa } \\
\text { Continua }\end{array}$ & Regla de Krimsky \\
\hline FORIA O TROPIA & $\begin{array}{c}\text { Desviación latente de } \\
\text { los ojos determina la } \\
\text { presencia o ausencia } \\
\text { de la habilidad fusional } \\
\text { motora }\end{array}$ & $\begin{array}{l}\text { Permite evaluar la presencia } \\
\text { y magnitud de una foria o } \\
\text { una tropia (estrabismo) en } \\
\text { un paciente. Una foria es } \\
\text { una desviación del ojo al } \\
\text { estar en reposo (latente). } \\
\text { Una tropia es una desviación } \\
\text { constante (manifiesta). } \\
\text { Examen a realizarlo el } \\
\text { oftalmólogo. }\end{array}$ & $\begin{array}{l}\text { Cualitativa } \\
\text { Nominal }\end{array}$ & $\begin{array}{l}\text { Cover Test (simple } \\
\text { y alternado) }\end{array}$ \\
\hline $\begin{array}{l}\text { AGUDEZA } \\
\text { VISUAL }\end{array}$ & $\begin{array}{c}\text { Es la capacidad de } \\
\text { resolución espacial del } \\
\text { sistema visual, se mide } \\
\text { a través de la cartilla de } \\
\text { Snellen que consta de } 11 \\
\text { filas de letras que van de } \\
\text { manera descendente para } \\
\text { así valorar la capacidad } \\
\text { visual del individuo. } \\
\text { Normal } 20 / 50-20 / 20 \\
\text { Subnormal } 20 / 60-20 \\
/ 200\end{array}$ & $\begin{array}{l}\text { La cartilla se coloca a } \\
\text { una distancia de } 6 \text { metros } \\
\text { en línea recta con el /la } \\
\text { adolescente a examinar, } \\
\text { procurando que sus ojos } \\
\text { se encuentren a la altura } \\
\text { de la línea verde de la } \\
\text { misma. Si el paciente sin } \\
\text { corrección (lentes) presenta } \\
\text { un valor mayor de } 20 / 20 \\
\text { se le considera emétrope } \\
\text { si es menor será amétrope. } \\
\text { Si utiliza corrección se le } \\
\text { denomina amétrope con } \\
\text { corrección }\end{array}$ & $\begin{array}{l}\text { Cualitativa } \\
\text { Nominal }\end{array}$ & Cartilla de Snellen \\
\hline EDAD & $\begin{array}{l}\text { Años de vida del } \\
\text { paciente }\end{array}$ & $\begin{array}{c}\text { Edad consignada en la ficha } \\
\text { de evaluación }\end{array}$ & $\begin{array}{l}\text { Cuantitativa } \\
\text { discreta }\end{array}$ & $\begin{array}{c}\text { Ficha de } \\
\text { evaluación }\end{array}$ \\
\hline SEXO & Identidad de la persona & & $\begin{array}{l}\text { Cualitativa } \\
\text { nominal }\end{array}$ & $\begin{array}{l}\text { Ficha de } \\
\text { evaluación }\end{array}$ \\
\hline
\end{tabular}

Agudeza visual: se define como la capacidad de percibir y diferenciar dos estímulos separados por un ángulo determinado (18). Se utilizó la cartilla de Snell, colocada a una distancia de 6 metros del adolescente evaluado, se consideró una visión optima los rangos de $20 / 1520 / 20 / 20 / 25$.
Cover test (cover- uncover y alternante): Es una herramienta de diagnóstico para la IC, se utiliza para descartar problemas estrábicos, permite evaluar la presencia de desviaciones de los ejes visuales y magnitud de una foria o una tropia en un paciente. Una foria es una desviación del ojo al estar en reposo 
(latente). Una tropia es una desviación constante (manifiesta).

Para evaluar la efectividad de la terapia se midió la disminución en el nivel de Insuficiencia de la Convergencia

El estudio constó de 3 fases: una fase inicial en la que se realizó la evaluación oftalmológica a 321 estudiantes previa invitación a los padres familia y a los estudiantes de los cuales 225 presentaron IC siendo la frecuencia de esta patología de $70,1 \%$. La segunda fase constó del tratamiento a 60 estudiantes a los que se aplicó: ejercicios de estiramiento y ejercicios oculomotores:

Procedimientos de los ejercicios oculomotores Evaluación Oftalmológica

Se realizó una primera evaluación que estuvo a cargo del oftalmólogo quien realizó el diagnóstico oftalmológico y la orden para que el adolescente realice los ejercicios oculomotores. Los resultados de las evaluaciones fueron anotados en una ficha de datos.

\section{Intervención}

Tomamos en cuenta para estos ejercicios las bases anatomofisiologicas del aprendizaje motor, el cual indica que mientras más información sea proporcionada al S.N.C. mayor será el nivel de desarrollo y organización neurológica, basándose en la velocidad de transmisión del estímulo generando una sumación espacial y temporal. Se realizó los ejercicios oculomotores a los adolescentes que cumplieron con los criterios de inclusión. Tuvo una duración de aproximadamente un mes y constó de 10 sesiones tres veces por semana, consistió en:

\section{Preparación: (5 $\mathrm{min})$}

Los investigadores aprendieron estimulación temprana pediátrica visual en el curso de métodos terapéuticos 2 , se procedió a realizar los ejercicios de estimulación propioceptivos según el procedimiento de Iñaki (14) se realizó la concientización de los movimientos oculares, constaron de pasar alrededor del ojo objetos con diferentes superficies (fibra esponja, corrospum, algodón y pelotas de mini-masaje). Luego se realizaron estiramientos que activaron los husos neuromusculares que disminuyen la fatiga de los músculos extraoculares. Estos estiramientos fueron cuatro: los dos primeros de pie, con los brazos elevados y el otro con los brazos juntos al cuerpo, se pidió al paciente que siga con la mirada el movimiento del dedo, los otros dos se realizaron en posición sedente con los brazos flexionados y aducidos al tronco, con las rodillas extendidas y la columna vertebral recta. Estos estiramientos fueron realizados por 20 segundos, con 3 repeticiones de cada serie.

\section{Ejercicios oculomotores: (20 min)}

En esta etapa los investigadores indicaron a los participantes trabajar cada ojo por separado, para igualar el funcionamiento entre ambos, enfatizando la estimulación del ojo menos hábil. Se realizó la obturación aleatoria y diversos ejercicios en las áreas del sistema oculomotor y acomodativo, aumentando el nivel de dificultad progresivamente y permitiendo la presencia de apoyos motores, cinestésicos o auditivos. Estos ejercicios fueron de la mano con coordinación ojo mano, memoria visual, percepción periférica, reconocimiento de formas y tamaños, espacio visual, etc.

Los ejercicios realizados fueron:

\section{Pelota de Marsden}

Se trabajó sentado durante 5 minutos, los investigadores colgaron la pelota en el techo a la altura de la nariz del paciente y a la distancia del brazo estirado. Se indicó a los participantes seguir la pelota en las diferentes direcciones sin movilizar la cabeza, seguidamente que nombre las letras que observe, esto se trabajó para desarrollar la visión periférica, las fijaciones y los seguimientos.

\section{Cuerdas de Brock}

Se trabajó sentado durante 5 minutos, se usó un cordón con cuatro bolas de colores. El investigador se colocó frente al participante sujetando un extremo del cordón, el paciente sujeta el otro extremo colocándolo bajo su nariz. Se colocó las cuatro bolas de colores a diferentes distancias, le indico al participante que sin mover la cabeza cambie la fijación de una bola a otra aleatoriamente. El investigador realizó 8 direcciones de mirada: estas direcciones fueron:1. Arriba a la derecha, 2. Arriba en el centro. 3. Arriba a la izquierda 4. A la izquierda,5. A la derecha, 6. Abajo a la derecha, 7. Abajo en el centro, 8. Abajo a la izquierda.

Esto se trabajó para enseñar a ver con ambos ojos simultáneamente, eliminar cualquier tendencia a 
suprimir un ojo y desarrollar la habilidad de cambiar la visión de un punto a otro.

\section{Tarjetas de Harts}

Se trabajó de pie durante 10 minutos con cartas de Hart para lejos y para cerca. Se realizo la obturación de cada ojo por separado mediante un parche. El investigador colocó en la pared una carta de letras grandes, se pidió al participante que se coloque a la máxima distancia donde pueda ver las letras y le entregó una carta pequeña al participante para que la coloqué a la altura de sus ojos. El investigador orientó y guió la manera de leer, 2 letras del cartel de lejos y 2 de la carta pequeña, leer una fila o columna de la carta de letras grandes y luego hacer lo mismo con la carta pequeña, leer de manera intercalada, se utilizó cartillas con letras y números de diferentes tamaños, se trabajó con barras $\mathrm{R} / \mathrm{V}$ en ambas cartas y unas gafas $\mathrm{R} / \mathrm{V}$ en el participante

\section{Lápices}

Se trabajó sentado, durante 5 minutos. Se utilizó lápices con imágenes, números y letras en cada carilla. El investigador sentado frente al participante colocó el lápiz a una distancia de $40 \mathrm{~cm}$ de los ojos del mismo, le indicó que siga el lápiz en diferentes direcciones, y que nombrara las letras, numero o imágenes que observe. El investigador utilizando dos lápices a la altura de los hombros del participante y a una distancia de $30 \mathrm{~cm}$, le indicó que nombre intercaladamente lo que observe sin mover la cabeza.

Estos ejercicios se realizaron para enseñar a ver con ambos ojos simultáneamente y eliminar cualquier tendencia a suprimir un ojo así como desarrollar la habilidad de cambiar la visión, con ambos ojos, de un punto a otro.

\section{Evaluación post-intervención}

Habiendo culminado y completado las sesiones de ejercicios se le volvió realizar las evaluaciones oftalmológicas. El resultado de esta segunda evaluación nos sirvió para comprobar el nuevo estado en que se encuentra el adolescente, comprobando si el ejercicio fue beneficioso o no.

Cuatro estudiantes se retiraron y solo 56 adolescentes cumplieron la terapia de ejercicios oculomotores. Y la tercera fase consistió, nuevamente, en la evaluación oftalmológica y se compararon los resultados obtenidos de ambas evaluaciones.

Se dio entrega tanto del consentimiento informado a los padres como del asentimiento informado a los adolescentes que cumplieron con todos los criterios de inclusión. Para el análisis estadístico se empleó frecuencias absolutas y relativas para las variables categóricas y para las variables numéricas medidas de tendencia central y de dispersión además para realizar la comparación antes y después de ejercicios oculomotores se empleó la prueba de Wilcoxon con un $\mathrm{p}$ de significancia de menor de 0,05 .

\section{RESULTADOS}

Se incluyeron 60 estudiantes con IC para recibir la terapia de estiramiento y ejercicios oculomotores, 4 de ellos se retiraron por inconvenientes con los horarios, molestias a la hora de recibir el tratamiento y no cumplir con la cantidad de sesiones requeridas; teniendo un total de 56 adolescentes que cumplieron la terapia.

De los 56 participantes en el estudio 19 (33,9\%) adolescentes del género femenino y $37(66,1 \%)$ fueron del género masculino, según año de estudio fueron de

Tabla 2. Niveles de convergencia antes y después de los ejercicios oculomotores según género en 3 instituciones educativas

\begin{tabular}{|c|c|c|c|c|c|c|c|c|c|c|c|c|}
\hline \multirow{3}{*}{ CONVERGENCIA } & \multicolumn{4}{|c|}{ Masculino } & \multicolumn{4}{|c|}{ Femenino } & \multicolumn{4}{|c|}{ Total } \\
\hline & \multicolumn{2}{|c|}{ Antes } & \multicolumn{2}{|c|}{ Después } & \multicolumn{2}{|c|}{ Antes } & \multicolumn{2}{|c|}{ Después } & \multicolumn{2}{|c|}{ Antes } & \multicolumn{2}{|c|}{ Después } \\
\hline & n & $\%$ & n & $\%$ & $\mathbf{N}$ & $\%$ & n & $\%$ & n & $\%$ & n & $\%$ \\
\hline NORMAL & 0 & 0 & 13 & 35,1 & 0 & 0 & 6 & 31,6 & 0 & 0 & 19 & 33,9 \\
\hline LEVE & 26 & 70,3 & 21 & 56,8 & 11 & 57,9 & 10 & 52,6 & 37 & 66,1 & 31 & 55,4 \\
\hline MODERADO & 9 & 24,3 & 3 & 8,1 & 8 & 42,1 & 3 & 15,8 & 17 & 30.4 & 6 & 10,7 \\
\hline ALTO & 2 & 5,4 & 0 & 0 & 0 & 0 & 0 & 0 & 2 & 3.5 & 0 & 0 \\
\hline TOTAL & 37 & 100 & 37 & 100 & 19 & 100 & 19 & 100 & 56 & 100 & 56 & 100 \\
\hline
\end{tabular}


primer año $4(7,1 \%)$, de segundo año $20(35,7 \%)$, de tercer año $14(25 \%)$, de cuarto año $9(16,1 \%)$ y de quinto año $9(16,1 \%)$. Del total de niños que realizaron

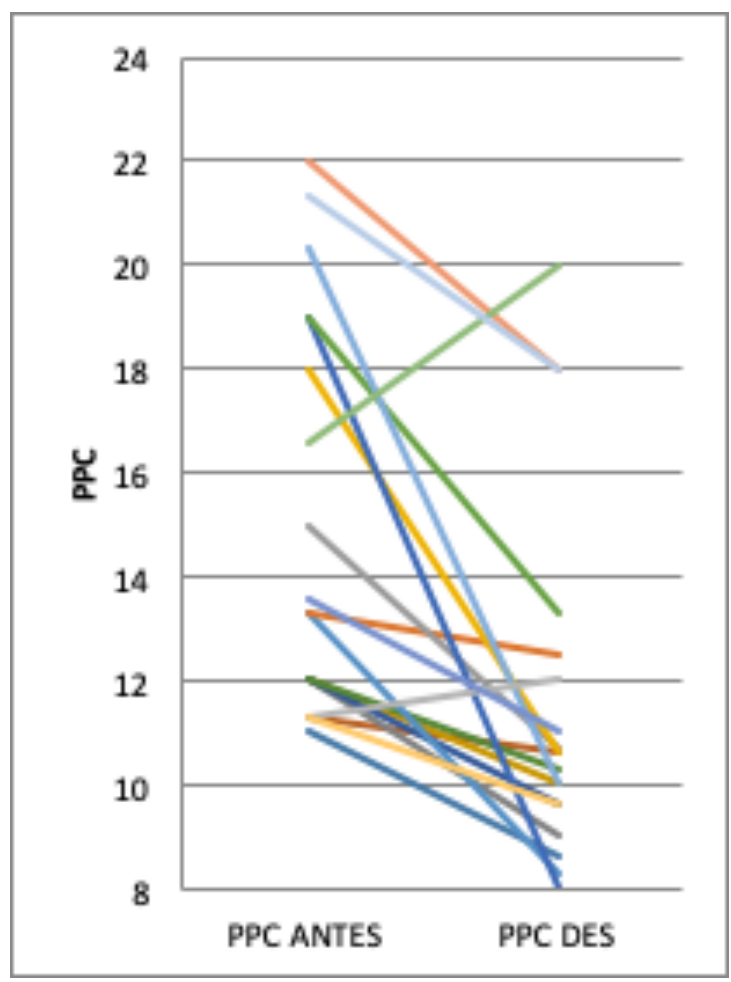

Gráfico 1. Niveles de PPC en adolescentes femeninos.

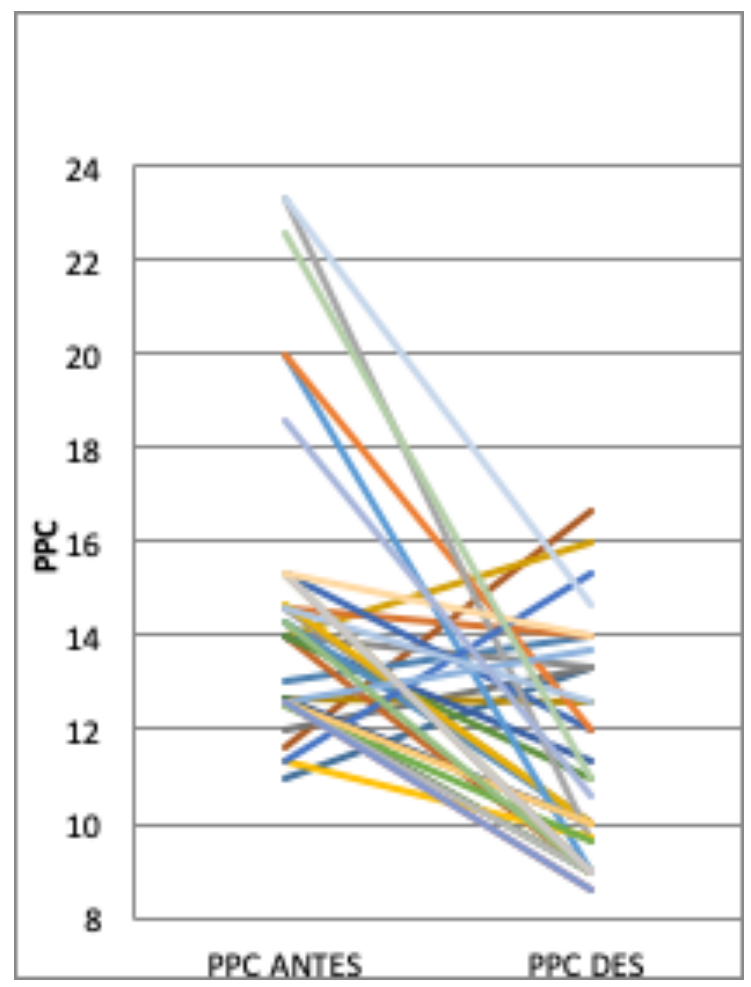

Gráfico 2. Niveles de PPC en adolescentes masculinos. la terapia $44(78,6 \%)$ fueron emétropes y $12(21,4 \%)$ amétropes con corrección. La IC leve fue la más frecuencia seguida del nivel moderado y por último el nivel alto siendo $66,1 \%, 28,6$ y $5,4 \%$ respectivamente, en el género masculino la IC leve fue de $70,3 \%$ muy superior al del grupo femenino. Después de los ejercicios oculomotores la convergencia normal fue de $33,9 \%$, el nivel leve bajó a 55,4\%, el nivel moderado a $10,7 \%$ y el nivel alto bajó a $0 \%$ (tabla 2 y gráficos 1,2 y 3$)$.

La puntuación de la IC con la regla de Krimsky en los adolescentes presento una media del 14,4 $\pm 1,4$ $\mathrm{cm}$. para el género femenino y $14,5 \pm 1,43 \mathrm{~cm}$ para el género masculino (tabla 3 ).

El puntaje del Punto Proximal de Convergencia (PPC) antes de la terapia fue en promedio de 14,8 $\mathrm{cm}(\mathrm{DS}=3,4)$ y después de la terapia disminuyó a $11,4 \mathrm{~cm}(\mathrm{DS}=2,77)$. La mediana de la puntuación de Punto Proximal de Convergencia (PPC) pre ejercicios oculomotores fue $14 \mathrm{~cm}$ (p25=12,6cm; - p75= $15,3 \mathrm{~cm})$ y post terapia fue $10,6 \mathrm{~cm}(\mathrm{p} 259,5 \mathrm{~cm}-\mathrm{p} 75$ $13,3 \mathrm{~cm})$ con diferencia estadísticamente significativa $(\mathrm{p}<0,03)$ (tabla 4).

Con respecto a la efectividad de la terapia 29 $(51,8 \%)$ de los pacientes que recibieron la terapia disminuyeron el nivel de IC, en mayor proporción mientras más severo fue el nivel de convergencia inicial: un 3(5,4\%) empeoró el nivel de IC y 24 $(42,8 \%)$ no tuvo variación de nivel (tabla 5 y gráfico $4)$.

\section{DISCUSIÓN}

La efectividad mide los beneficios obtenidos por una aplicación en condiciones reales. Las medidas de efectividad no son tan universales como la eficacia, sino que su generalización depende, en cierto grado, de la población a la que se aplica y de las condiciones de su aplicación (19).

En las revisiones de la base científica de Pubmed en el año de 1999 aparece una de los primeros estudios relacionado con la eficacia de la terapia visual en una población adulta siendo un ensayo clínico en el que participaron sesenta hombres adultos mayores de 40 años (edad media, 65 años) donde la intervención tuvo un éxito en $61,9 \%$ de los pacientes que recibieron terapia en el consultorio, el $30 \%$ de los pacientes que recibieron terapia en casa solamente, y en el 10,5\% del grupo control (20). En el año 2001 
se describe el uso de ortópticos para el tratamiento de la IC, no especificando el material usado sino basándose solamente en ejercicios de punto cercano o estimulación de convergencia ocular (21).
Si bien la frecuencia de IC es variable (10), sobretodo leve e infrecuente en el nivel alto (17), el beneficio de la terapia de ejercicios oculomotores observado es en todos los grupos, y es en el último grupo donde encontramos mayor efectividad.

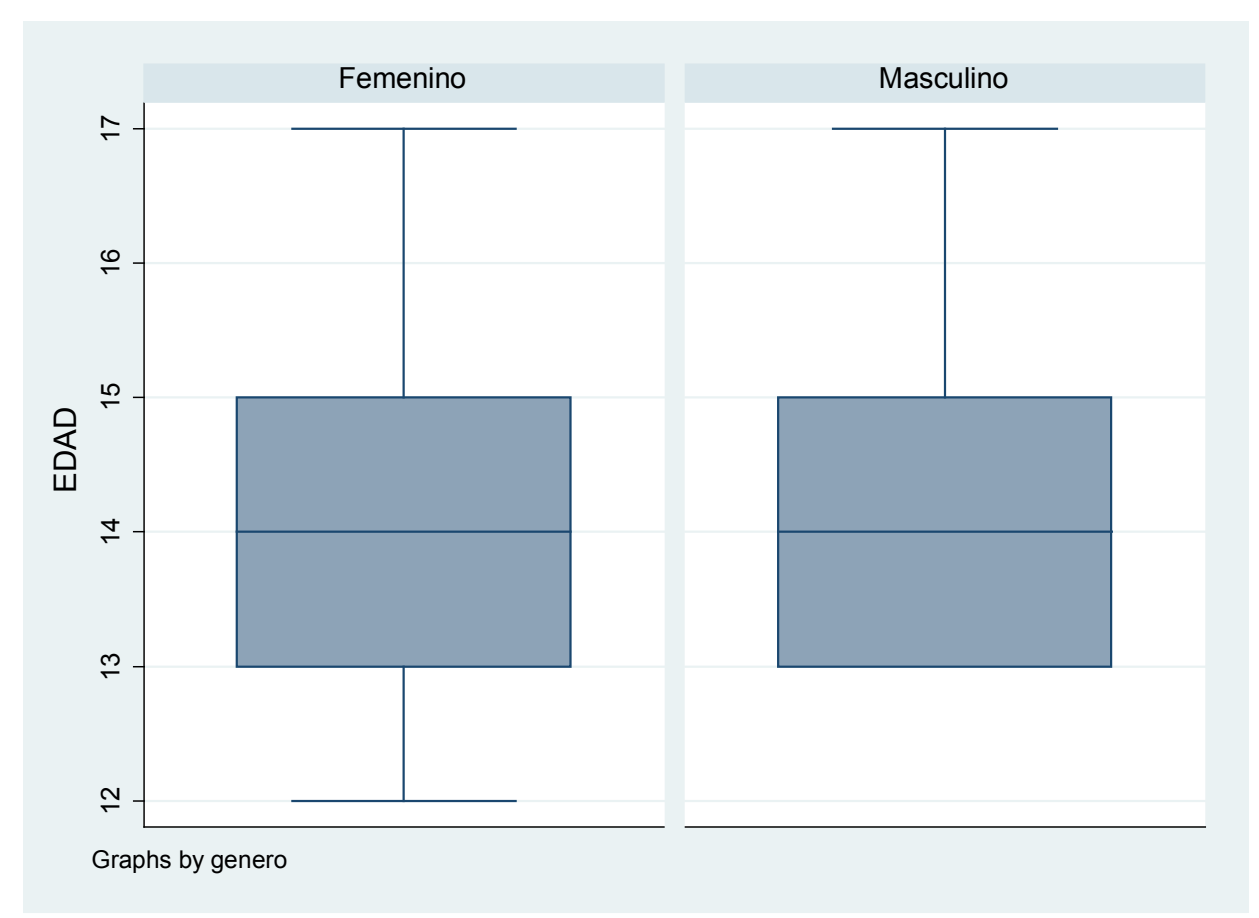

Gráfico 3. Edad según género en 3 instituciones educativas

Tabla 3. Edad según género en estudiantes con IC en 3 instituciones educativas

\begin{tabular}{lccccc}
\hline \multicolumn{1}{c}{ Género } & Media & Desv. Estd. & Percentil 25 & Percentil 50 & Percentil 75 \\
\hline Femenino & 14,2 & 1,36 & 13 & 14 & 15 \\
Masculino & 14,5 & 1,43 & 13 & 14 & 15 \\
Total & 14,4 & 1,39 & 13 & 14 & 15 \\
\hline
\end{tabular}

Tabla 4. Punto Proximal de Convergencia antes y después de los ejercicios oculomotores en 3 instituciones educativas

\begin{tabular}{cccccc}
\hline PPC & Media & Desv. Estd. & Percentil 25 & Percentil 50 & Percentil 75 \\
\hline Antes & 14,8 & 3,4 & 12,6 & 14 & 15,3 \\
Después & 11,4 & 2,7 & 9,5 & 10,6 & 13,3 \\
\hline
\end{tabular}

Tabla 5. Variación de niveles de IC después de los ejercicios oculomotores

\begin{tabular}{lrrrrccccc}
\hline \multirow{2}{*}{$\begin{array}{c}\text { Niveles de IC } \\
\text { convergencia inicial }\end{array}$} & \multicolumn{2}{c}{$\begin{array}{c}\text { IC final } \\
\text { Normal }\end{array}$} & \multicolumn{2}{c}{$\begin{array}{c}\text { IC final } \\
\text { Leve }\end{array}$} & \multicolumn{2}{c}{$\begin{array}{c}\text { IC final } \\
\text { Moderado }\end{array}$} & $\begin{array}{c}\text { IC final } \\
\text { Alto }\end{array}$ \\
\cline { 2 - 10 } & $\mathbf{n}$ & $\mathbf{\%}$ & $\mathbf{n}$ & $\mathbf{\%}$ & $\mathbf{n}$ & $\mathbf{\%}$ & $\mathbf{n}$ & $\mathbf{\%}$ \\
\hline Leve & 13 & 35,1 & 21 & 56,8 & 3 & 8,1 & - & - \\
Moderado & 5 & 29,4 & 9 & 52,9 & 3 & 17,7 & - & - \\
Alto & 1 & 50,0 & - & - & 1 & 50,0 & - & - \\
\hline
\end{tabular}




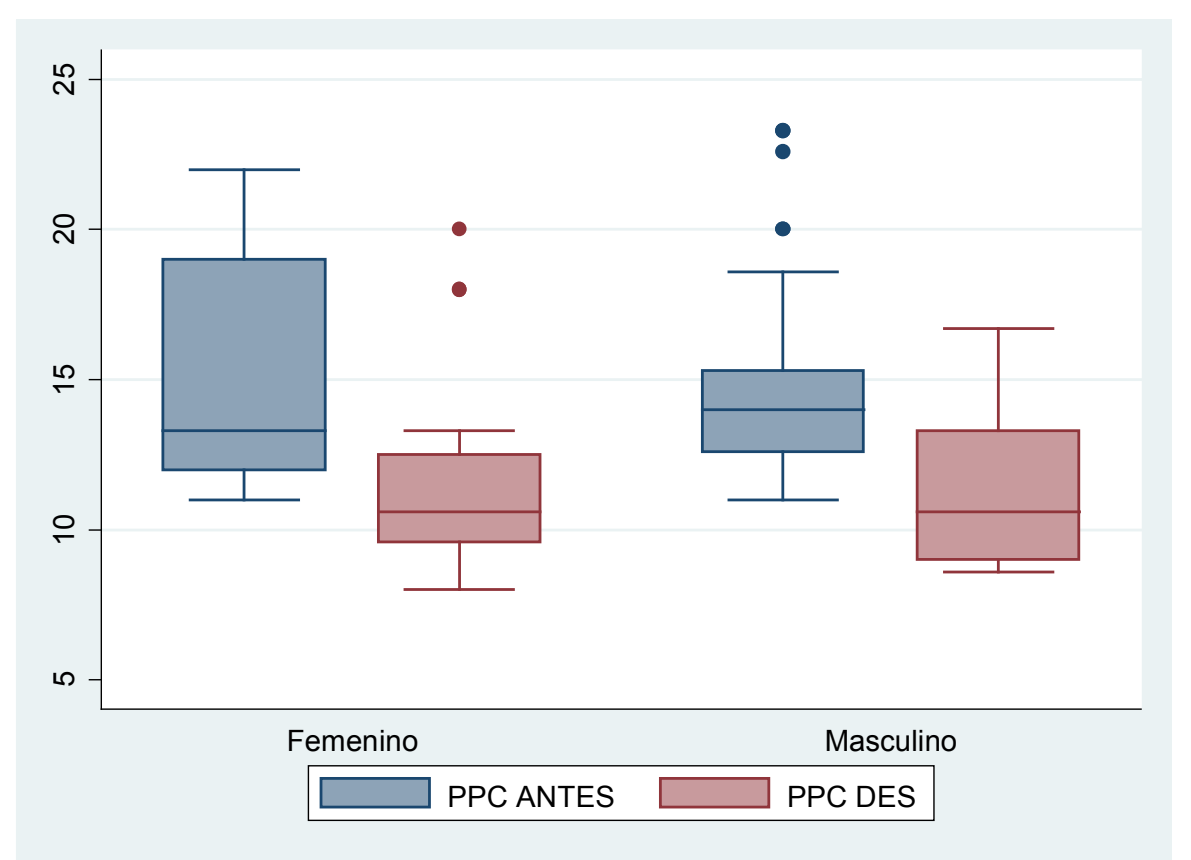

Gráfico 4. Niveles de PPC antes y después de los ejercicios Oculomotores según género en 3 instituciones educativas.

Nuestros resultados coinciden con los estudios realizados en España y Bogota $(10,17)$, donde con un número mayor de sesiones logran mayor mejoría de la IC. Asimismo los estudios de Berrojo (10) y Dragomir et al., obtuvieron mejores resultados en el grupo que realizó ejercicios en cada sesión y adicionalmente en casa, en comparación a quienes solo realizaron ejercicios en la sesión de terapia programada (21).

El desarrollo de los ejercicios oculomotores es multidisciplinario e involucra a médicos oftalmólogos, optómetras y fisioterapeutas, este conjunto de profesionales de la salud (16). Este grupo puede abarcar patologías sensoriales y motoras oculares en la población, y aunque en nuestro medio esta área aún no tiene una intervención global para el área educativa; la importancia de la detección de estas patologías o síndromes radica en su relación con trastornos de aprendizaje, y se recomienda en casi todos los casos terapia visual (21).

En conclusión, nuestros resultados muestran que los ejercicios oculomotores favorecen a la corrección de la insuficiencia de convergencia, por lo cual recomendamos incluir como parte de la intervención terapéutica apoyarse en áreas de estimulación visual de centros especializados en rehabilitación neurológica (7).

\section{REFERENCIAS BIBLIOGRÁFICAS}

1. Salgado C. Ambliopía y estrabismo. Tesis. Santiago: Pontificia Universidad Católica de Chile; 2005; 31-36 pp.

2. Molina NP, Foreo C. Insuficiencia de Convergencia. Ciencia y tecnología para la salud visual y ocular. 2010; 2: 97.

3. Lorea J. Caracterización de insuficiencia de convergencia en niños de 6 a 12 años de edad de la población escolar de la escuela Netzahualcóyotl, en el municipio de Hermosillo sonora. Tesis doctoral. Ciudada de Mexico: Instituto Politecnico Nacional; 2012.

4. Gila L, Villanueva A, Cabeza R. Physiopathology and recording techniques of ocular movements. An Sist Sanit Navar. 2009; 32(3): 9-26.

5. Dellosso L, Daroff R. Características y técnicas de registro de los movimientos oculares. Neurooftalmología. Barcelona: Salvat; 1982.

6. Hall J. Compendio de fisiología médica. Barcelona, España: Elveiser; 2011.

7. Rodríguez S. Anatomía de los órganos del lenguaje, visión y audición.. Madrid, España: Médica Panamericana; 2003.

8. Snell R. Neuroanatomía Clínica. Buenos Aires: Médica Panamericana; 2007.

9. Rodríguez M, López M, Sánchez M. Diagnóstico sobre alteraciones de los Movimientos Oculomotores $(\mathrm{MOM})$, con pruebas de medición subjetiva en niños entre 7 a 9 años con problemas de lectura y bajo 
rendimiento escolar en dos colegios de Bogotá. Ciencia y Tecnología para la salud Visual y Ocular. 2006; 6: 13-23.

10. Berrojo I, Escolar M. Terapia Visual en la Escuela. Madrid: XIII Master en Optometría y Entrenamiento visual; 2002.

11. Becerra V. Valoración de la acomodación en pacientes miopes pre y post cirugía refractiva láser técnica Lasik. Bogota: Universidad de la Salle. Bogotá; 2009.

12. McGregor M. Convergence Insufficiency and Vision Therapy. Pediatr Clin N Am. 2014; 61. 621-630.

13. Arnoldi K, Reynolds D. A review of convergence insufficiency: what are we really accomplishing with exercises. American Orthoptic Journal. 2007; 57: 123-30.

14. Iñaki P. Terapia Manual en el sistema oculomotor técnicas avanzadas para la cefalea y trastornos del equilibrio. Barcelona: Editorial Elveiser Masson; 2012.

15. Pilat A. Terapias Miofasciales: Inducción Miofascial. Madrid: Editorial McGraw-Hill España; 2003.

16. Márquez M. Análisis de la situación de Salud del INO. Lima: Instituto Nacional de Oftalmología; 2010.
17. Ramírez P. Efectividad del tratamiento ortóptico de insuficiencias de convergencia realizado a pacientes entre 13 y 25 años del IIo entre enero y octubre del 2007. Tesis. Bogota: Universidad de la Salle. Facultad de Optometría; 2008.

18. Quezada O. Efecto del tratamiento con anti-vegf sobre la agudeza visual en pacientes con maculopatía diabética. Tesis de Grado. Trujillo, Perú: Universidad de Trujillo; 2016.

19. Ministerio de Sanidad y Consumo - Instituto de Salud "Carlos III" Agencia de Evaluación de Tecnologías Sanitarias (AETS). Evaluación Epidemiológica de Tecnologías de salud. Madrid: AETS Instituto de Salud Carlos III; 1995.

20. Birnbaum MH, Soden R, Cohen AH. Efficacy of vision therapy for convergence insufficiency in an adult male population. Am Optom Assoc. 1999; 70 (4): 134-139.

21. Dragomir M, Trus L, Chirilá D, Stingu C. Orthoptic treatment efficiency in convergence insufficiency treatment. Oftalmología. 2001; 53 (3): 72-78. 